\title{
Answer to the letter to the editor of Y. Li et al. concerning "Intraosseous basivertebral nerve ablation for the treatment of chronic low back pain: a prospective randomized double-blind sham-controlled multi-center study" by Fischgrund JS, et al. [Eur Spine J; (2018) 27(5): 1146-1156]
}

\author{
Jeffrey S. Fischgrund ${ }^{1}$
}

Received: 2 July 2019 / Accepted: 2 July 2019 / Published online: 9 August 2019

(C) The Author(s) 2019

We thank the reader for expressing interest in our clinical trial of the Intracept procedure that treats chronic back pain in patients with evidence of endplate pathology (Modic changes) via ablation of the basivertebral nerve (BVN) within affected lumbar and sacral vertebrae. We appreciate the knowledgeable comments on our study and are happy to have an opportunity to clarify our protocol and rationale for the readers of the European Spine Journal.

In the SMART study, the BVN ablation target was the terminus of the basivertebral foramen (the nutrient channel seen on clinical imaging), typically midline and between 40 and $60 \%$ of posterior to anterior distance across the vertebral body. This location was chosen based on anatomic data published for L4, L5 and S1 in 2011 and 2016. The reader is correct that these cadaveric studies describe different vertebral nerve entry points and trajectories between L4 and L5 (posterior border with a posterior-anterior path) and S1 (lateral border with a lateral-medial path). While this is true, in both cases the nerves cluster at the vertebral centrum (anatomic center) then branch to the cranial and caudal endplates where they contribute to vertebrogenic pain symptoms arising at sites of endplate pathology. As a result, the BVN ablation target did not differ between L3, L4, L5 and $\mathrm{S} 1$ vertebral bodies. Indeed, subset analyses show no outcome differences between patients, where S1 was or was not included in the treated vertebral body levels. Consequently, there is no evidence suggesting that different BVN targeting

Jeffrey S. Fischgrund

jsfischgrund37@gmail.com

1 Oakland University William Beaumont School of Medicine, 3535 West 13 Mile Road, Suite 744, Royal Oak, MI 48073, USA should have been implemented at S1 and would have led to improved outcomes.

Regarding the treatment effect, the study's primary endpoint, the mean change in ODI at 3 months, was -20.5 points in the treatment arm, as compared to -15.2 points in the sham $\operatorname{arm}(p=0.019$, per-protocol population). Analysis of the treatment arm at 24 months also demonstrated a statistically significant reduction in baseline in ODI of 23.5 points $(53.7 \%)$ and VAS of 3.59 points (52.9\%). As with other studies comparing sham to an invasive active treatment for a condition with pain as the primary complaint, the sham response was both robust and enduring. Nevertheless, the improvement in the treatment arm from baseline was clinically meaningful and was consistent with a second randomized controlled trial comparing BVN ablation to standard care that showed a -25.3 point mean change in ODI at 3 months, compared with a -4.4 point improvement in the standard care arm.

In summary, we believe that our study design and results support the hypothesis that a significant source of pain in CLBP patients is vertebrogenic, and that BVN ablation can be a safe and effective approach to improve clinical outcomes in this challenging patient population.

Open Access This article is distributed under the terms of the Creative Commons Attribution 4.0 International License (http://creativecommons.org/licenses/by/4.0/), which permits unrestricted use, distribution, and reproduction in any medium, provided you give appropriate credit to the original author(s) and the source, provide a link to the Creative Commons license, and indicate if changes were made.

Publisher's Note Springer Nature remains neutral with regard to jurisdictional claims in published maps and institutional affiliations. 